\title{
Erratum
}

\section{Synthesis of Functionalized $p$-Terphenyls Based on Site-Selective Suzuki Cross-Coupling Reactions of Bis(triflates) of 2,5-Dihydroxybenzoate}

Muhammad Nawaz, Muhammad Farooq Ibad, Obaid-Ur-Rahman Abid, Rasheed Ahmad Khera, Alexander Villinger, Peter Langer* Synlett 2010, 150.

The advance online publicaton (e-First) version of this article contained errors in References 15 and 17.

Those errors have been corrected for both the current online version and the print publication. 